\author{
A. KOHLSTEDT ${ }^{1}$ \\ S. KALBFLEISCH ${ }^{1}$ \\ T. SALDITT ${ }^{1, \bullet}$ \\ M. REICHE ${ }^{2}$ \\ U. GÖSELE ${ }^{2}$ \\ E. LIMA $^{3}$ \\ P. WILLMOTT ${ }^{4}$
}

\title{
Two-dimensional $X$-ray waveguides: fabrication by wafer-bonding process and characterization
}

\author{
${ }^{1}$ Institute for X-ray Physics, Georg-August University Göttingen, Friedrich-Hund-Platz 1, \\ 37077 Göttingen, Germany \\ 2 Department 2, Max Planck Institute of Microstructure Physics, Weinberg 2, 06120 Halle, Germany \\ 3 ESRF, 6 rue Jules Horowitz, BP220, 38043 Grenoble Cedex, France \\ 4 SLS, PSI, 5232 Villigen PSI, Switzerland
}

\section{Received: 30 November 2007/Accepted: 10 December 2007 Published online: 26 January 2008 • () The Author(s) 2008}

ABSTRACT The fabrication of two-dimensionally confining $\mathrm{X}$-ray waveguides enables the generation of nanoscopic X-ray beams. First applications of such waveguides for lens-less holographic imaging have already been demonstrated, but were limited by the fabrication methods and the design. To overcome these limitations, we present here the fabrication process for a second generation of X-ray waveguide with air or vacuum as guiding channel, based on e-beam lithography, ion etching and subsequent wafer bonding. This is a first step towards waveguides fulfilling requirements of high transmission and high confinement, since the process can be scaled down to smaller channel dimensions from the present structures. We address the structuring method used and present results of first X-ray characterization at synchrotron beamlines, under two entirely different beam settings, corresponding to the coupling of a coherent beam and an incoherent beam.

PACS 41.50.th; 42.82.Cr; 07.85.Qe

\section{$1 \quad$ Introduction}

The use of X-rays for the investigation of condensed matter structure relies on the precise definition of $\mathrm{X}$-ray beams, which are then used in scattering, imaging or spectroscopy experiments. Since high-resolution lenses in particular for hard X-rays are not available, most modern synchrotron beamlines and laboratory instruments use slits or pinholes to define the cross section of the X-ray beams. As the scattering volume or the sample size decreases in many applications to the nanometer range, beam definition at the nanoscale presents an entirely new challenge for X-ray science and technology. Already today, many imaging, spectroscopy and lithography experiments require the use of very small apertures (also called spatial filters in some microscopy techniques) with a cross section far in the sub-micron range for X-ray-beam definition. The spatial resolution of these techniques is limited by the cross-sectional dimensions of the available apertures; see for example lens-less X-ray Fourier transform holography [1] or proximity X-ray lithography [2]. In the limit of smaller and smaller cross sections, the beam-

Fax: +49-551-39-9430, E-mail: tsaldit@gwdg.de defining apertures must change in geometric proportion from two-dimensional apertures to channels of high aspect ratio, since the absorption requires a minimum thickness which is then much larger than the lateral dimension, in particular as the photon energy $E$ increases.

The optics of such structures is no longer given by absorption masks as known from standard diffraction theory, but is much better described by waveguiding with a discrete set of modes, as described in [3-5]. In practice, the fabrication of ultra-narrow apertures or channels presents the most difficult task. Some possible realizations of ultra-small pinholes or slits are planar waveguides with a tunable air gap [6,7], thinfilm structures $[8,9]$ and pinholes fabricated using focused ion beams [10]. The first two-dimensional waveguides were presented in [11] with poly-methyl-methacrylate (PMMA) as core material with a width $w$ between 50 and $300 \mathrm{~nm}$ covered by a 6-nm-thick Cr cladding. The beam was then coupled in through the thin cladding layer, according to the so-called scheme of resonant beam coupling (RBC). The disadvantage of this coupling scheme is the presence of strong reflected and transmitted beams, and the low photon flux exiting from the channel. To improve the optical setup and to make it suitable for imaging applications, the incident beam was pre-focused by bent mirrors and coupled directly into the front side of the waveguide [12]. In this case, the primary beam and radiative modes are absorbed in the cladding, while the guided wave propagates through the waveguide core. This scheme was realized based on a polymer channel defined by e-beam lithography (EBL) and covered by silicon.

In contrast to polymer channels covered by high-density material, which served as the first generation of two-dimensional X-ray waveguide channels, the optical requirements of high transmission and high confinement of the beam now push towards the use of air/vacuum as a guiding layer. The main advantage of air (or vacuum) is the reduction of absorption losses and beam damage. Furthermore, the difference of the refractive indices between core and cladding is increased, i.e. the confining potential can become higher. Finally, growth of materials may lead to entirely different interfacial roughness than etching of highly perfect single crystals. In summary, waveguide channels in semiconductor materials such as silicon or germanium are highly desirable, but demand elaborate structuring methods, combining EBL, reactive ion etching (RIE) and wafer bonding (WB), which is used in the 
last step to cover and seal the channels. Here we will denote $\mathrm{X}$-ray waveguides fabricated by EBL, RIE and WB as secondgeneration X-ray waveguides.

Wafer bonding has previously been used by Poulsen et al. to fabricate one-dimensional X-ray waveguides with a cross section of $100 \mu \mathrm{m} \times 74 \mathrm{~nm}$ [16]. We have also used the bonding process previously to cover an array of waveguide channels which could, however, not be addressed separately. Furthermore, the bonding process was not completely satisfactory [13]. In this work we present well-separated single channels located at the interior interface of two bonded wafers. However, in this study, only the fabrication of single and separated channels with a rather large cross section in the range of a few microns was achieved, for technical and instrumental reasons, described below. It is clear that, in the future, the presented fabrication method can be scaled down for X-ray waveguide channels with a cross section in the range of $10-100 \mathrm{~nm}$, which will be the next step. The fabrication method is described in Sect. 2, while Sect. 3 presents the first X-ray results obtained at two synchrotron beamlines, one with essentially laterally coherent, the other with incoherent (focused) X-ray radiation. The results show that in the coherent case even a broad channel can be used to obtain large divergence of the exit beam, due to a structured near-field intensity distribution in the channel.

\section{Fabrication of wafer-bonded waveguides}

X-ray waveguides consist of two materials with different refractive indices $n_{j}=1-\delta_{j}+\mathrm{i} \beta_{j}$. $\delta_{j}$ is the refractiveindex decrement $\left(10^{-5}-10^{-8}\right.$ for hard X-rays) and $\beta_{j}$ is the imaginary part of the refractive index responsible for the absorption in the material [14]. The wave propagates in the guiding material with the smaller $\delta$ in a defined number $N$ of modes given by the solution of the Helmholtz equation [15]. Former X-ray waveguides used a polymeric guiding core which absorbed a part of the radiation and also suffered damage caused by the X-ray beam. Air as guiding material avoids these disadvantages. The basic idea of the design for such a new generation of two-dimensional X-ray waveguides consists in creating narrow trenches on the nm scale by EBL, to transfer them into silicon by etching processes and finally to close them with a second silicon wafer in a bonding process. There have already been achievements in fabrication of

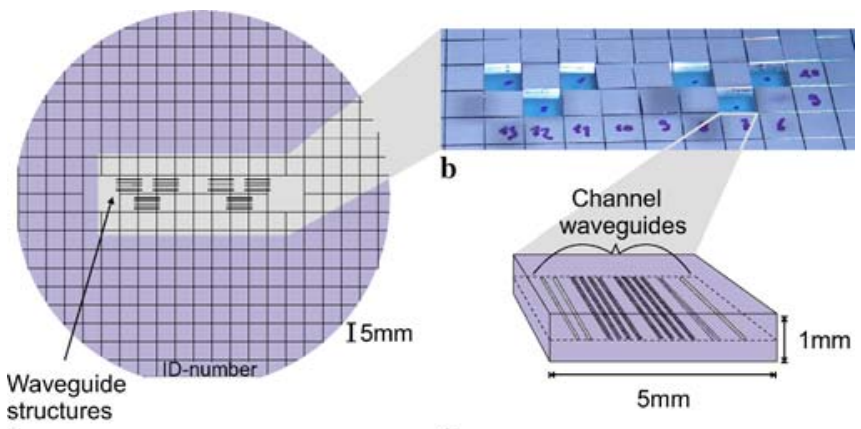
structures

FIGURE 1 (a) Schematic showing the positioning of the lithographic structures on a 4-in silicon wafer. (b) Photograph of the bonded and sawn silicon wafers. Some of the waveguide chips have already been taken out of the marked gaps for use. (c) Schematic of a completed X-ray waveguide chip

one-dimensional X-ray waveguides by UV photolithography, with a lower limit in the lateral width of $1.5 \mu \mathrm{m}$ [16]. The use of e-beam lithography permits the creation of structures on the nm scale and therefore the fabrication of multi-modal and mono-modal two-dimensional waveguides [13].

\section{$2.1 \quad$ Structuring process}

For fabrication, double-polished, $500 \pm 25 \mu \mathrm{m}$ thick, 4-in Si(100) wafers provided by the MPI of Microstructure Physics in Halle were used. On both sides a $5 \times 5 \mathrm{~mm}^{2}$ grid with $200-\mu \mathrm{m}$-wide lines was etched approximately $2-\mu \mathrm{m}$ deep into the surface. This grid served as orientation for the alignment of the e-beam lithography patterns on the wafer as shown in Fig. 1a.

The wafers were cleaned using the so-called RCA1 and RCA2 method, labeled according to the David Sarnoff Research Center [17]. Then, a system of etching masks was deposited on the wafers; see Fig. 2. The wafers already possessed a native 18-nm-thick oxide layer covering the silicon surface. As a second layer, 20-nm chromium was evaporated with an electron-beam evaporator (UNIVEX 450, Leybold). On top of this layer, a 100-nm-thick poly-methylmethacrylate (PMMA) layer was spin coated to form a positive e-beam resist [13]. To this end, a solution of $2 \mathrm{~g}$ Elvacite 2041 (Lehmann+Voss) in $100 \mathrm{ml}$ 2-methoxy-ethyl-acetate (8.06061, MERCK) was used. Multiple lithography patterns were combined to form a standard array. Several quadrants
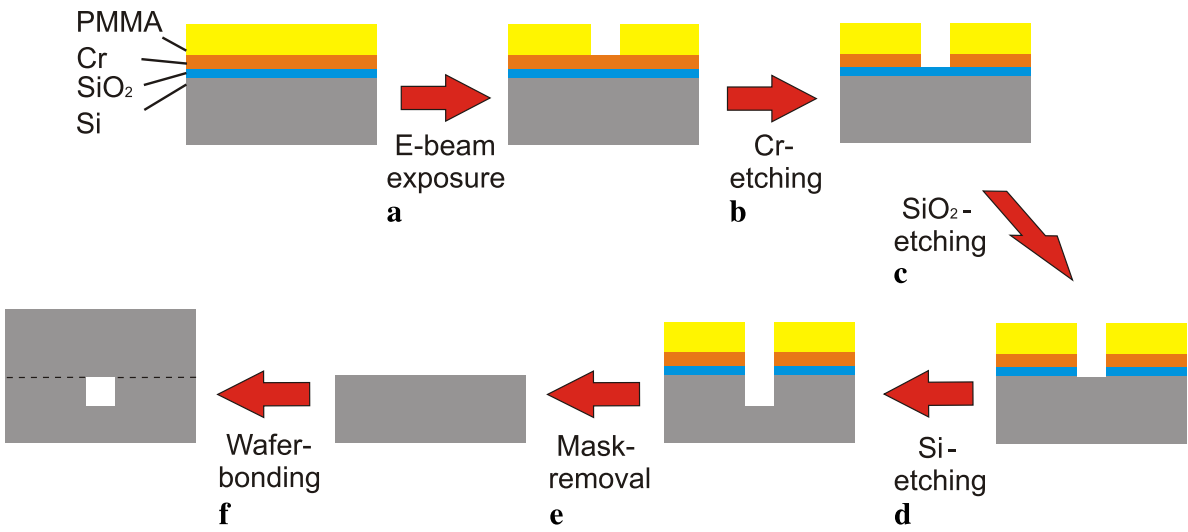

FIGURE 2 Sketch of the structuring process used. (a) Exposure and developing of the ebeam resist. (b) Transfer of the pattern into the chromium layer via RIE process. (c) Etching of the $\mathrm{SiO}_{2}$ in BHF. (d) Transfer of the waveguide trench into the silicon wafer via RIE process. (e) Removal of all etch masks. (f) Bonding a second $\mathrm{Si}$ wafer on the structured one to cover the waveguide core 


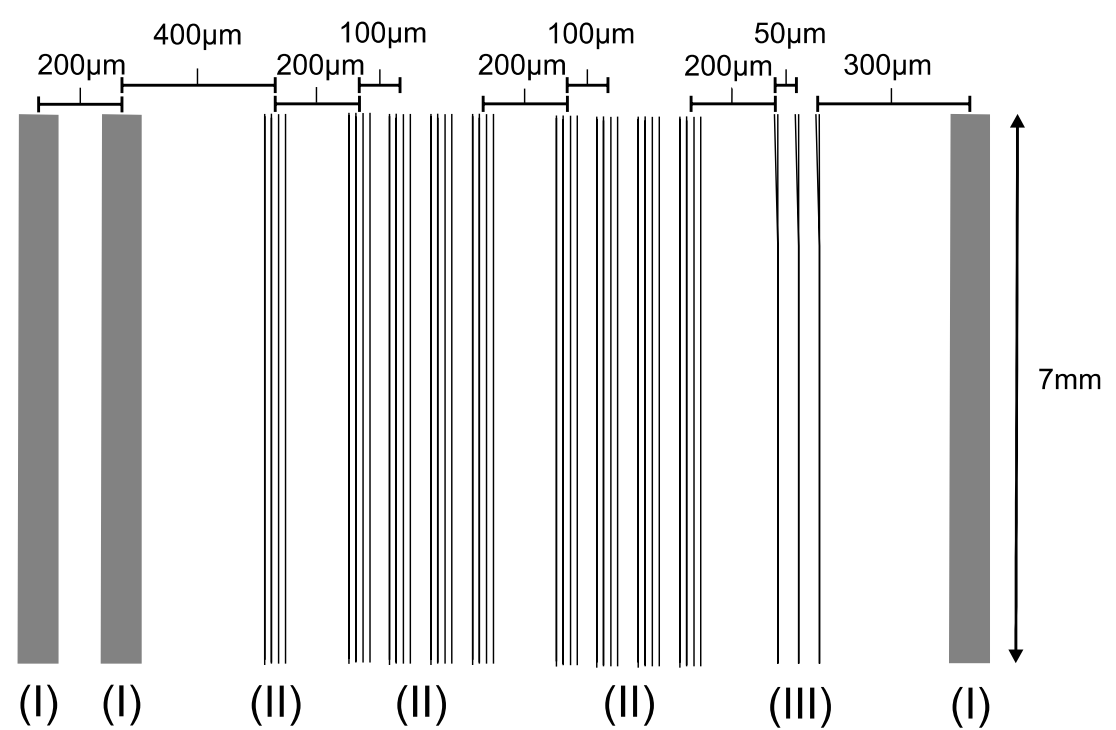

were processed in parallel, each with the same array of patterns for later use. Importantly, the individual channels in each array were spaced wide enough so that the channels could be addressed individually. The arrays were aligned with respect to the grid and were exposed with the e-beam system LION LV1 (Leica). They were composed of different channel and grating patterns, each with a length of $7 \mathrm{~mm}$; see Fig. 3. The 100- $\mu \mathrm{m}$-wide gratings with a period of $400 \mathrm{~nm}$ served as benchmark for X-ray-beam alignment on each waveguide array. Several groups each of four single lines with varying width $(90-170 \mathrm{~nm})$ were placed in between the gratings, as well as three Y-shaped channels with different openings $(5,7.5$ and $10 \mu \mathrm{m})$. The Y-shaped waveguides consist of a pair of two lines which are close together at the front side, but which diverge towards the exit side of the waveguide chip. Y-shaped waveguides can be used to split up the incoming beam for off-axis (reference-beam) holography [18]. The exposed resist was developed in a $4: 1$ mixture of ethyleneglycolmonoethylether (8.01554, MERCK) and ethyleneglycolmonobutylether (8.00857, MERCK) (Fig. 2a).

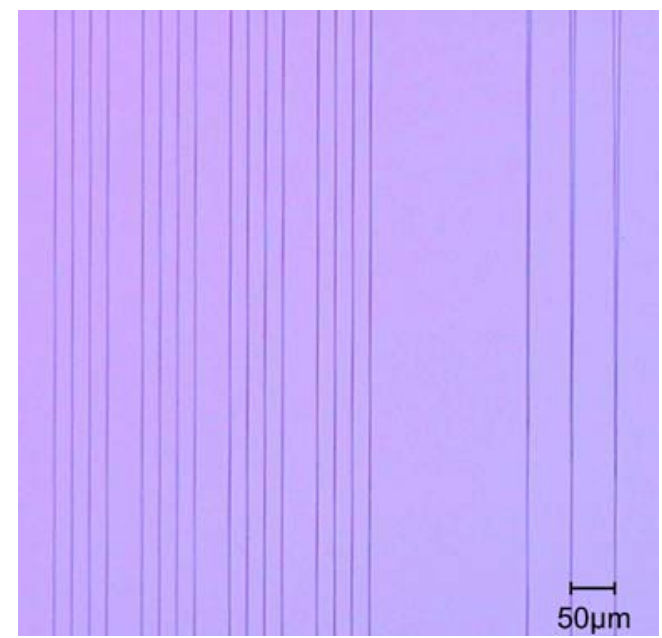

FIGURE 4 Micrograph of the structures transferred in silicon taken with a light optical microscope (ZEISS)
FIGURE 3 Design of one lithographic pattern with the different types of structures: (I) $100-\mu \mathrm{m}$-wide gratings with a period of $400 \mathrm{~nm}$, (II) groups of four single lines with varying width $(90-170 \mathrm{~nm})$, (III) Y-shaped structures with different exit openings (5, 7.5 and $10 \mu \mathrm{m})$
The structures were transferred into the chromium layer using a reactive ion etch (RIE) (Oxford Plasma Lab System 100) with the etch gases $\mathrm{Cl}_{2}$ and $\mathrm{O}_{2}$ (Fig. 2b). To etch the $\mathrm{SiO}_{2}$, a $1: 6: 14 \mathrm{BHF}$ solution of $50 \% \mathrm{HF}, 40 \% \mathrm{NH}_{4} \mathrm{~F}$ and ultra-pure water was used (Fig. 2c). The last structuring step was performed using the RIE with the etch gases $\mathrm{SF}_{6}$ and $\mathrm{O}_{2}$ to transfer the waveguide trenches into the silicon wafer (Fig. 2d). The PMMA was removed with acetone, the chromium was removed using the etch solution Selectipur (MERCK) and the oxide was removed in a BHF bath (Fig. 2e). A lightmicroscopy image of the structures obtained after all etching steps is shown in Fig. 4.

\subsection{Wafer bonding and completion of waveguide chips}

All etching processes described above were performed in a class 1 cleanroom at the MPI of Microstructure Physics, to achieve the necessary cleanliness for a successful bond process. Microscopic roughness and macroscopic planarity of the wafers are important parameters for bonding. Note that any dust particle will introduce a defect, preventing bonding at least in a defect zone of radius $R$. A limit for the waviness or height distortions by particles or defects is given by a relation of the lateral extension $R$ of a non-bonded (defect) area and the depth $h$ of the defect zone arising from waviness [19]. In the case of large defects and small wafer thickness $(R>2 d)$ the condition $h<R^{2} / \sqrt{2 E^{\prime} d^{3} /(3 \gamma)}$ must be fulfilled for bonding, while in the case of thick wafers and small-scale defects $(R<2 d)$ the critical height deviation is $h<3.6 \sqrt{R \gamma / E^{\prime}}$. For this consideration, both wafers are assumed to have the same thickness $d, \gamma$ is the surface energy and Young's modulus $E$ and Poisson's ratio $v$ define the parameter $E^{\prime}=E-v$.

Therefore, the structured wafer was again cleaned using the RCA1 and RCA2 method and then bonded to an unstructured silicon wafer of the same type. The process was carried out in a microcleanroom (SÜSS Microtech) at room temperature in air. A lightly exerted pressure on the center of the wafers starts the broadening of the bonding force. The thus developed van der Waals bonds transform into covalent 


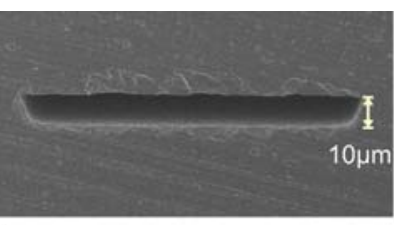

a

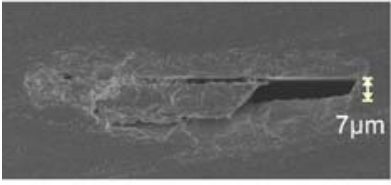

b

FIGURE 5 Scanning electron micrographs of the waveguide front side recorded by the LION LV1 (Leica). (a) A 100- $\mu$ m-wide channel formed by percolation of grating channels. (b) A channel entrance blocked by a silicon particle. (c) Smaller channels obtained from single lines. The lines of $170 \mathrm{~nm}$ had been exposed by EBL, but larger channels were formed by under-etching

bonds during an annealing process. To this end, the bonded wafers were baked out in an argon-rinsed vacuum oven for $5 \mathrm{~h}$ at $950{ }^{\circ} \mathrm{C}$.

Finally, the wafers were cut along the grid to a length of $5 \mathrm{~mm}$ with a wafer saw (saw blade: $30 \mu \mathrm{m}$, DISCO DAD 3220), see Fig. $1 \mathrm{~b}$ and c. Figure 5 shows SEM micrographs of the waveguide entrance sides. The gratings were over-etched so that $100-\mu \mathrm{m}$-wide and $7-\mu \mathrm{m}$ - to $10-\mu \mathrm{m}$-high trenches were produced; see Fig. 5a and b. Figure 5c shows four single lines with a resulting cross section of $4 \mu \mathrm{m} \times 3 \mu \mathrm{m}$. Some entrances were blocked by silicon particles which were detached during the sawing of the 4-in wafer. This underlines the necessity to fabricate several channels in one chip, which can then be tested subsequently during the synchrotron beamtime.

\section{3}

\section{First X-ray characterization}

The bonded waveguides have been tested in two synchrotron-radiation experiments. In the experiment described first, a laterally coherent undulator beam was used. In the experiment described second, a focused incoherent wiggler beam was coupled into the waveguides. The coherent coupling was carried out at the ID10C undulator beamline of the ESRF in Grenoble [21], which provides high brilliance, high coherence and a flux density of the unfocused primary beam of $5 \times 10^{13}$ photons/s at a ring current of $150 \mathrm{~mA}$. The beam energy was adjusted by a double-bounce $\mathrm{Si}(111)$ monochromator to $10.44 \mathrm{keV}$. Higher harmonics were suppressed by double-bounce silicon mirrors inclined at $0.16^{\circ}$ incidence angle located $56.8 \mathrm{~m}$ from the insertion device.

Using the comparably high flux density of the beamline the unfocused undulator beam was coupled into the front of the waveguide adjusted behind a pair of beam-defining slits. Figure 6 shows a scan across the entrance side of the waveguide array described in Sect. 2. The groups of four single lines are clearly identified. Adjusting on one single waveguide (IV.4, shown in the SEM image in Fig. 5) the coherent far field resulting from multi-modal beam propagation in the wave-

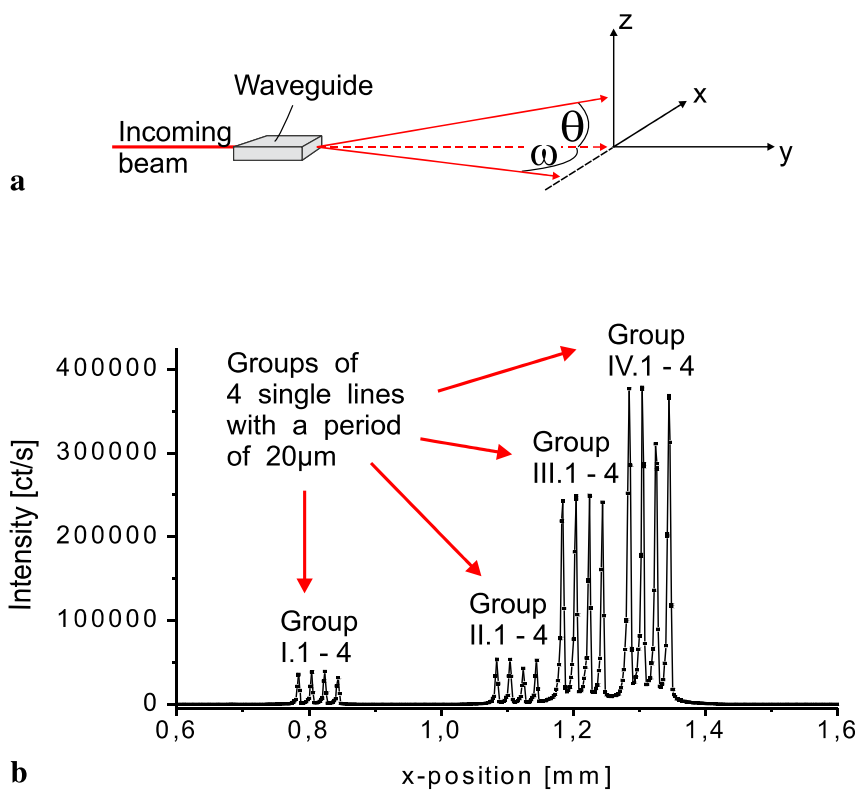

FIGURE 6 (a) Schematic setup to record the waveguide far-field pattern. (b) Scan across the entrance side for alignment of a bonded waveguide array. It shows four groups, each of four single lines with varying width and a period of $20 \mu \mathrm{m}$

guide was recorded with a back-illuminated CCD (Princeton, ROPER Scientific SX1300) with a pixel size of $20 \times 20 \mu \mathrm{m}^{2}$ (see Fig. 7). It was placed at a distance of $2700 \mathrm{~mm}$ behind the waveguide. The recorded far-field pattern is strongly speckled with an envelope which can be described by a Gaussian; see Fig. 7. The full width at half maximum (FWHM) of the Gaussian fits (envelope functions) of the slices are $\Delta \omega=0.0659^{\circ}$ in the horizontal and $\Delta \theta=0.0394^{\circ}$ in the vertical direction,
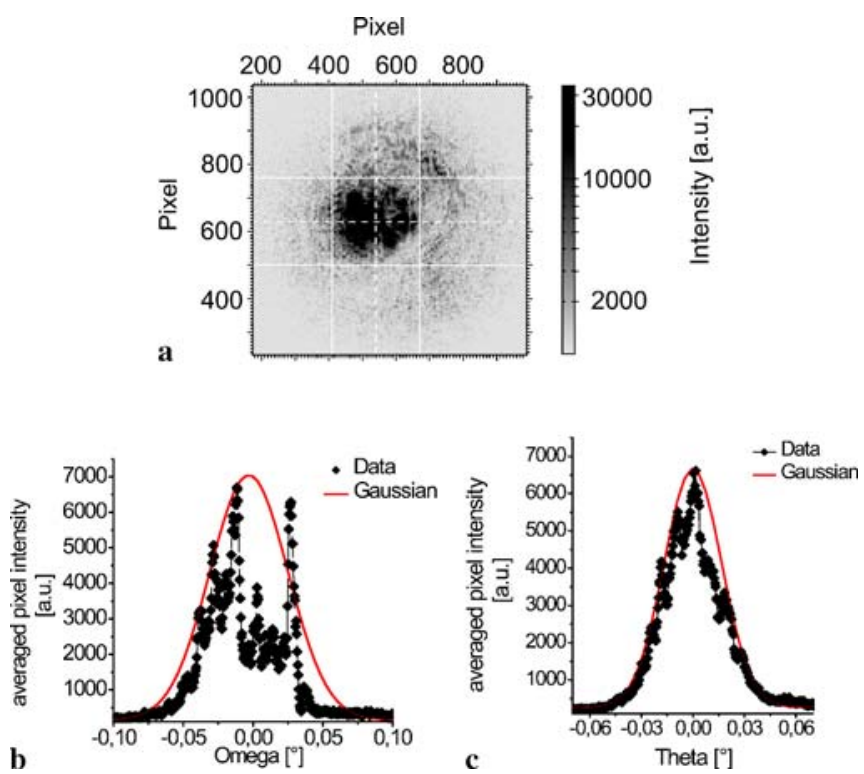

FIGURE 7 (a) CCD image of the waveguide far field (multi-modal waveguide channel IV.4), obtained at the ID10C beamline of the ESRF in Grenoble. The crossed lines mark the areas for the slices shown in (b) and (c). The far-field pattern shows a strong speckled structure corresponding to the farfield transform of a near-field intensity distribution. (b) and (c) Horizontal and vertical slices through the far field fitted by a Gaussian curve to define an envelope function 

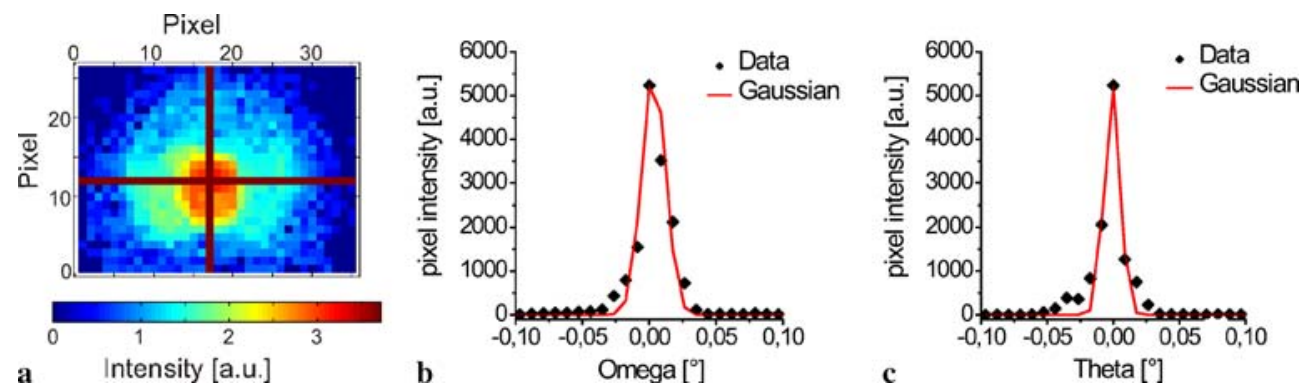

FIGURE 8 (a) Image of the far-field pattern recorded with the Pilatus II pixel detector at the material science beamline at the SLS. The slice positions through the peak region of the far field are marked with crossed lines and plotted at the right as pixel intensity versus horizontal (b) and vertical (c) pixels, respectively. The slices are fitted to Gaussians to obtain the angular width of the beam (FWHM)

respectively. In reciprocal space this corresponds to $\Delta q_{\mathrm{horz}}=$ $0.0061 \AA^{-1}$ and $\Delta q_{\text {vert }}=0.0036 \AA^{-1}$, respectively. Note that the static speckle pattern observed corresponds to the farfield transform of a near-field intensity distribution which may arise from interference due to defects in the channel and mode mixing.

The incoherent experiment was performed at the material science beamline of the Swiss light source (SLS) at PSI, Villigen [22]. The radiation is generated by a wiggler source and it is therefore not as brilliant and characterized by a much smaller spatial coherence as compared to the undulator beam of the experiment described above. The flux density was $1 \times 10^{13}$ photons $/ \mathrm{s}$ in a focused beam of $500 \times 500 \mu^{2}$, at a photon energy of $13.5 \mathrm{keV}$, selected by a fixed-exit doublecrystal monochromator. The monochromator was placed in between two mirrors used for higher-harmonic rejection and focusing. Each mirror consisted of a 1-m-long silicon blank, coated with rhodium, which could be tilted by up to $5 \mathrm{mrad}$ and bent $(5 \mathrm{~km}<R<30 \mathrm{~km})$ as required for the desired photon energy [24].

The waveguide was placed on a hexapod (PI, Karlsruhe, Germany) behind the entrance slits $\left(0.03 \times 0.03 \mathrm{~mm}^{2}\right)$. The far-field pattern was measured with a Pilatus II pixel detector (SLS Detector Group) [23]. With a pixel size of $172 \times$ $172 \mu \mathrm{m}^{2}$ and a sample-detector distance of $1116 \mathrm{~mm}$, one pixel was equivalent to an angle of $\Delta_{\text {pixel }}=0.0088$. The far field of the waveguide channel (IV.4) described above now is much smoother and not speckled as in the coherent case. Along the main symmetry axis, the measured width (FWHM, Gaussian fits) was $\Delta \omega=0.0207^{\circ}$ in the horizontal and $\Delta \theta=$ $0.0141^{\circ}$ in the vertical direction, respectively, see Fig. 8. In reciprocal space this corresponds to $\Delta q_{\text {horz }}=0.0025 \AA^{-1}$ and $\Delta q_{\text {vert }}=0.0017 \AA^{-1}$, respectively.

Comparing the results of the two experiments, we see that the far-field width (after transformation from angular to reciprocal space to obtain values independent of photon energy), the width of the far-field pattern is almost three times higher in the coherent case. Note that the lateral coherence length at the ID10C beamline exceeds the cross section of the waveguide entrance, while it is not the case in the focused wiggler beam. Hence, an essentially completely coherent wave is coupled into the multi-modal channel at the ID10C beamline while the opposite is true for the material science beamline of SLS. In the coherent case, the width of the far field is much higher than expected from the geometric cross section. Considering a simple slit-like diffraction [25] according to the equation
$I \propto w^{2} \frac{\sin ^{2}\left(\frac{\pi w}{\lambda} \sin \omega\right)}{\left(\frac{\pi w}{\lambda} \sin \omega\right)^{2}} h^{2} \frac{\sin ^{2}\left(\frac{\pi h}{\lambda} \sin \theta\right)}{\left(\frac{\pi h}{\lambda} \sin \theta\right)^{2}}$,

with $\lambda$ defining the particular wavelength, a rectangular channel of width $w=4 \mu \mathrm{m}$ and height $h=3 \mu \mathrm{m}$ would result in a far-field intensity distribution with a width (FWHM) of $\Delta q_{\text {horz }}=1.5 \times 10^{-4} \AA^{-1}$ and $\Delta q_{\text {vert }}=1.9 \times 10^{-4} \AA^{-1}$. For a waveguide the far field is given by the Fraunhofer diffraction pattern [26]. In small-angle approximation the intensity for a two-dimensionally confining waveguide is given as a function of $\theta$ and $\omega$; compare Fig. 6:

$I(\theta, \omega) \propto\left|\int \Psi(x, z) \exp (\mathrm{i} k \theta z) \exp (\mathrm{i} k \omega x) \mathrm{d} y \mathrm{~d} z\right|^{2}$.

It thus represents the square modulus of the Fourier transform of the field $\Psi(x, z)$ [27] at the end face of the waveguide. We see that the measured far fields in both cases are about one order of magnitude higher than the resulting intensity distribution behind a rectangular slit, also taking into account that the cross section of the waveguide is only approximately rectangular; see Fig. 5. Particularly in the coherent case we examine the wave-propagation properties of a waveguide, which transmits a highly coherent and slightly divergent X-ray beam.

\section{Summary and conclusion}

We have described the fabrication process of twodimensional X-ray waveguides with air as guiding layer, fabricated via e-beam lithography, reactive ion etching and wafer bonding. The obtained waveguide channels have been tested at two synchrotron-radiation sources proving their optical properties. The results show that the coherent far field is much broader than expected from a simple calculation based on homogeneous illumination. Most likely, the interference and mixing of multiple modes and the deviations from an ideal channel structure (roughness, waviness) lead to a 'structured' near field with intensity variations on length scales which are smaller than the waveguide cross section. For future applications, the fabrication scheme discussed above should be scaled towards a smaller channel cross section, which should be easily feasible if the resists, etching masks and parameters are adapted, including the use of an e-beam resist with a better plasma etching stability. To warrant a better protection of the wafer surface during the RIE process and to keep the exposed lines narrow, one should use an e-beam resist 
and thickness which are designed for higher plasma etching stability. If available, it could be deposited directly onto the oxide layer to reduce the number of plasma processes and achieve smaller two-dimensional waveguides. To clean the channel entrance from unwanted particles caused by the sawing, one can use a focused ion beam, which has already been used to polish a waveguide front side [20]. In conclusion, we have fabricated separated single channels located at the interior interface of two bonded wafers, which demonstrates that lateral structuring by lithography and reactive ion etching is fully compatible with wafer bonding, and can be used for advanced X-ray optical devices. The first X-ray results indicate that the resolution of a waveguide source in imaging may not be limited by the channel width, if the interior field distribution becomes sharper and has smaller length scale components due to interference. We consider this work as a first step for the fabrication and use of second-generation X-ray waveguides.

ACKNOWLEDGEMENTS We acknowledge SLS and ESRF for synchrotron beamtime and excellent working conditions, and we thank Franz Pfeiffer for additional help and advice during the SLS experiment, as well as Jens Seeger for his help in the early fabrication process. Support by the Deutsche Forschungsgemeinschaft through SFB755 'Nanoscale Photonic Imaging' is gratefully acknowledged.

OPEN ACCESS This article is distributed under the terms of the Creative Commons Attribution Noncommercial License which permits any noncommercial use, distribution, and reproduction in any medium, provided the original author(s) and source are credited.

\section{REFERENCES}

1 S. Eisebitt, J. Lüning, W.F. Schlotter, M. Lörgen, O. Hellwig, W. Eberhardt, J. Stöhr, Nature 432, 885 (2004)

2 S. Knaack, J. Eddington, Q. Leonard, F. Cerrina, M. Onellion, Appl. Phys. Lett. 84, 3388 (2004)

3 C. Fuhse, C. Ollinger, S. Kalbfleisch, T. Salditt, J. Synchrotron. Radiat. 13, 69 (2006)

4 C. Fuhse, T. Salditt, Appl. Opt. 45, 4603 (2006)
5 D. Pelliccia, I. Bukreeva, A. Cedola, S. Lagomarsino, Appl. Opt. 45, 2821 (2006)

6 M.J. Zwanenburg, J.F. van der Veen, H.G. Ficke, H. Neerings, Rev. Sci. Instrum. 71, $1723(2000)$

7 M.J. Zwanenburg, J.H.H. Bongaerts, J.F. Peters, D.O. Riese, J.F. van der Veen, Phys. Rev. Lett. 85, 5154 (2000)

8 C. Fuhse, A. Jarre, C. Ollinger, J. Seeger, T. Salditt, R. Tucoulou, Appl. Phys. Lett. 85, 1907 (2004)

9 W. Jark, A. Cedola, S. Di Fonzo, M. Fiordelisi, S. Lagomarsino, N.V. Kovalenko, V.A. Chernov, Appl. Phys. Lett. 78, 1192 (2001)

10 S. Eisebitt, M. Lörgen, W. Eberhardt, J. Lüning, S. Andrews, J. Stöhr, Appl. Phys. Lett. 84, 3373 (2004)

11 F. Pfeiffer, C. David, M. Burghammer, C. Riekel, S. Salditt, Science 297, 230 (2002)

12 C. Ollinger, C. Fuhse, A. Jarre, T. Salditt, Physica B 357, 53 (2005)

13 A. Jarre, J. Seeger, C. Ollinger, C. Fuhse, C. David, T. Salditt, J. Appl. Phys. 101, 054306 (2007)

14 J. Als-Nielsen, D. McMorrow, Elements of Modern X-Ray Physics (Wiley, New York, 2001)

15 D. Marcuse, Theory of Dielectric Optical Waveguides (Academic, New York, London, 1974)

16 M. Poulsen, F. Jensen, O. Bunk, R. Feidenhans'1, D.W. Breiby, Appl. Phys. Lett. 87, 261904 (2005)

17 W. Kern, Handbook of Semiconductor Wafer Cleaning (Noyes, Park Ridge, NJ, 1993)

18 C. Fuhse, C. Ollinger, T. Salditt, Phys. Rev. Lett. 97, 254801 (2006)

19 U. Gösele, Q.-Y. Tong, A. Schumacher, G. Kräuter, M. Reiche, A. Plöß1, P. Kopperschmidt, T.H. Lee, W.-J. Kim, Sens. Actuators 74, 161 (1999)

20 C. Ollinger, A Waveguide-based Lens-less X-ray Microscope, Dissertation, Universität Göttingen (2006)

21 Beamline ID10C at the ESRF in Grenoble, http://www.esrf.eu/UsersAndScience/Experiments/SCMatter/ID10A/ BeamlineDescription/\#troikaIII

22 B.D. Patterson, R. Abela, H. Auderset, Q. Chen, F. Fauth, F. Gozzo, G. Ingold, H. Kühne, M. Lange, D. Maden, D. Meister, P. Pattison, T. Schmidt, B. Schmitt, C. Schulze-Briese, M. Shi, M. Stampanoni, P.R. Willmott, Nucl. Instrum. Methods A 540, 42 (2005)

23 Material science beamline of the Swiss light source at the PSI Villigen, http://sls.web.psi.ch/view.php/beamlines/ms/sd/pixel/index.html

24 Material science beamline of the Swiss light source at the PSI Villigen, http://sls.web.psi.ch/view.php/beamlines/ms/layout/optics/index.html

25 H. Niedrig, in Lehrbuch der Experimentalphysik, Band 3, Optik, ed. by L. Bergmann, C. Schaeffer (Walter de Gruyter, Berlin, New York, 2004)

26 M. Born, E. Wolf, Principles of Optics, 6th edn. (Pergamon, Oxford, 1987)

27 C. Fuhse, T. Salditt, Opt. Commun. 265, 140 (2006) 